\title{
A comparison of two methods for measuring thermal thresholds in diabetic neuropathy
}

\author{
DAVID LEVY, RALPH ABRAHAM, GORDON REID \\ From the Department of Diabetes and Endocrinology, Central Middlesex Hospital, Acton Lane, Park Royal, \\ London
}

SUMMARY Thermal thresholds can be measured psychophysically using either the method of limits or a forced-choice method. We have compared the two methods in 367 diabetic patients, 128 with symptomatic neuropathy. The Sensortek method was chosen for the forced-choice device, the Somedic modification of the Marstock method for a method of limits. Cooling and heat pain thresholds were also measured using the Marstock method. Somedic thermal thresholds increase with age in normal subjects, but not to a clinically significant degree. In diabetics Marstock warm threshold increased by $0.8^{\circ} \mathrm{C} /$ decade, Sensortek by $0.1{ }^{\circ} \mathrm{C} /$ decade. Both methods had a high coefficient of variation in normal subjects (Sensortek 29\%, Marstock warm 14\%, cool 42\%). The prevalence of abnormal thresholds was similar for both methods (28-32\%), though Marstock heat pain thresholds were less frequently abnormal (18\%). Only $15-18 \%$ of patients had abnormal results in both tests. Sensortek thresholds were significantly lower on repeat testing, and all thresholds were higher in symptomatic patients. Both methods are suitable for clinical thermal testing, though the method of limits is quicker. In screening studies the choice of a suitable apparatus need not be determined by the psychophysical basis of the test.

Electrophysiology has been the most widely used method for measuring peripheral nerve function in diabetes, but even in its most sophisticated forms, ${ }^{1}$ it assesses function in only the largest and most heavilymyelinated nerve fibres.

There is much interest in small fibres particularly in view of their role in the painful symptoms of diabetic neuropathy. Several authors ${ }^{2-4}$ have suggested that small-fibre function is affected preferentially in diabetic neuropathy, though there is no definite evidence for this view. At the moment the only peripheral small fibre modality which can be routinely assessed is thermal sensitivity using psychophysical methods. Many devices are now available for testing this modality. One, the Marstock method, ${ }^{5}$ uses the method of limits, the others a forced-choice method. The latter is generally preferred as it is thought to reduce response bias $^{67}$ and hence increase reliability and precision. The purpose of the study, therefore, was to compare two thermal testing methods, one forced-

Correspondence to: Dr David Levy, Department of Diabetes and Endocrinology, Central Middlesex Hospital, Acton Lane, Park Royal, London NW10 7NS, United Kingdom.

Received 7 March 1989.

Accepted 19 May 1989 choice, the other employing a method of limits, in large number of diabetic subjects.

\section{Methods}

All tests were carried out on the right foot. Thermodes were lightly applied to the skin over the dorsolateral aspect of the foot. Foot temperature was maintained at $30-32^{\circ} \mathrm{C}$.

(1) Forced-choice: Sensortek method ${ }^{8}$

This and some other forced-choice methods measure "static" thermal sensitivity; alternative forms of the apparatus have been described. ${ }^{4}$ The patient is asked to identify the warmer of two Peltier-controlled elements, differing in temperature by set amounts, by moving his foot from one plate to the other. One of the elements is held at a standard reference temperature $\left(30^{\circ} \mathrm{C}\right)$ and the temperature of the other randomly varied above or below this (range $0 \cdot 1-20^{\circ} \mathrm{C}$ ). The responses are plotted on a graph and the temperature difference altered according to the pattern of correct and incorrect responses, described as the up-down-transformation rule ${ }^{9}$ (UDTR). Several variants of the UDTR have been described; here the thermal threshold was taken as the mean of six points at which the plot changes either from an up-to-down or a down-to-up direction. In normal and nonneuropathic patients the test takes about 20 minutes, but in neuropathic patients the increased time required to change the temperature of the plates between stimulus pairs can extend the test to $\mathbf{4 0}$ minutes. This limitation applies to all thermal threshold methods. 
(2) Method of limits: Somedic modification of "Marstock" method $^{5}$

This apparatus measures dynamic thermal sensitivity. Warm and cool stimuli are delivered separately to a single Peltiercontrolled thermode attached to the foot. Increasing the rate of rise of temperature allows heat pain thresholds to be measured. The power settings for the current studies were $25 \%$ (of maximum heat output) for warm and cool thresholds and $60 \%$ for the heat pain thresholds. The thermode temperature is plotted continuously on a chart recorder. The device uses the method of limits, in which the patient activates a hand held switch when warmth or coolness is perceived. This reverses the current and brings the thermode temperature back to reference level $\left(32^{\circ} \mathrm{C}\right.$, adjustable). A safety cut-out temperature is set (in this study $50^{\circ} \mathrm{C}$ ) to minimise the risk of thermal injury. Consecutive stimuli are delivered at random intervals and the mean response amplitude is recorded as the limen. Ten stimuli were used for warm and cool thresholds, 5 for the heat pain threshold. The total time for measuring three thresholds on each limb is approximately 20 minutes. Standardisation of thermode application pressures and calibration of the Marstock apparatus to give quantitative values for the rate of heat delivery were not used in the current study.

\section{Subjects}

All diabetic patients 66 years of age or under attending our clinics were invited for thermal testing. All 576 who attended had Sensortek thermal thresholds measured and 367 both Sensortek and Marstock warm thresholds. Their clinical characteristics are shown in the table. During the initial part of the study, Marstock heat pain thresholds were not measured in 57 patients. The comparative study tests were usually carried out on the same day by the same operator; 131 patients had repeat Sensortek tests one to 12 weeks after the first. One hundred and twenty eight patients were considered to have symptomatic neuropathy on the basis of the presence of one or more of the following: symmetrical lower limb numbness, paraesthesiae, contact sensitivity, spontaneous pain or burning. Reference ranges for the Sensortek and Marstock methods were established in a group of 78 healthy hospital personnel (age range 18-67). The usual exclusion criteria were observed for normal subjects, that is, the presence of neurological signs, symptoms, or known neurological disease; alcohol intake $>14$ units per week; drug therapy or untreated endocrine disease which could interfere with peripheral nerve function.

The intrasubject variability of the four methods was assessed by testing non-diabetic subjects on three occasions over a period of three to six weeks. The studies were approved by the Ethics Committee of Brent Health Authority.

Table Clinical characteristics of the 367 diabetic subjects tested with both thermal threshold methods (mean SD)

\begin{tabular}{lc}
\hline Age & $50 \cdot 9$ SD $11 \cdot 1$ years (range 18-66) \\
Time since diagnosis & $8 \cdot 1$ SD $8 \cdot 0$ years (range $1-47)$ \\
Gender & Male $211(57 \%)$. Female $156(43 \%)$ \\
Type I (insulin-dependent) & $63(17 \%)$ \\
Currently insulin treated & $112(31 \%)$ \\
Symptomatic neuropathy & $128(35 \%)$ \\
\hline
\end{tabular}

Statistics

Data are presented as mean and SD, with numbers of subjects in parentheses. The thermal thresholds were logarithmically transformed for the regressions. Statistics comprised Spearman correlation coefficients, multivariate general analysis and Mann-Whitney $U$ test for independent samples.

\section{Results}

\section{(1) Normal subjects}

(a) Sensortek method Mean thermal threshold was $0.35, \mathrm{SD} 0.30^{\circ} \mathrm{C}(78)$. With this number of subjects, there was no significant change in thermal sensitivity with age $(\mathrm{r}=0.12, \mathrm{p}=0.31)$.

(b) Somedic method Mean warm threshold was 5.0 SD $1.8^{\circ} \mathrm{C}(78)$, cool threshold $1.9 \mathrm{SD} 1.4^{\circ} \mathrm{C}(78)$, heat pain threshold $11.7 \mathrm{SD} 2.4^{\circ} \mathrm{C}(78)$. There was a small but statistically significant deterioration in all thresholds with age (warm $r=0.48, p<0.001$; cool $r=0.23, p=0.05$; heat pain $r=0.34, p=0.002$ ). The slopes of the regressions give the following changes in ${ }^{\circ} \mathrm{C} /$ decade: warm threshold $0.7^{\circ} \mathrm{C}$, cool threshold $0.2^{\circ} \mathrm{C}$, heat pain $0.4^{\circ} \mathrm{C}$.

(c) Variability In five normal subjects tested on three occasions over a period of six weeks, there was a mean coefficient of variation (CV) of $29 \%$ for the Sensortek method. The Marstock method had a mean CV for warm threshold of $14 \%$ and for cool threshold $42 \%$, though the CV for warm threshold could be reduced to approximately $8 \%$ by taking the mean of ten responses after omitting the first ten.

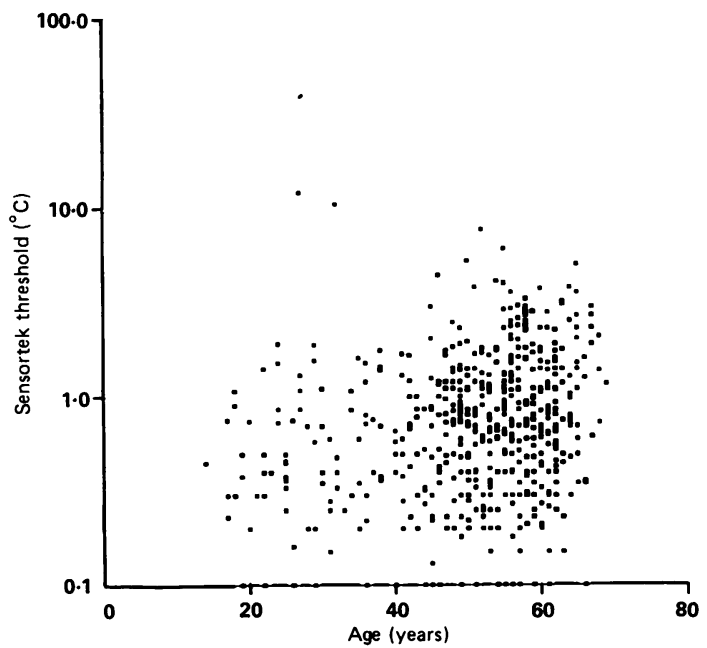

Fig 1 Scattergram of relationship between age and Sensortek thermal thresholds (logarithmic scale) in 576 diabetic subjects. $r=0.25, p<0.001$. 


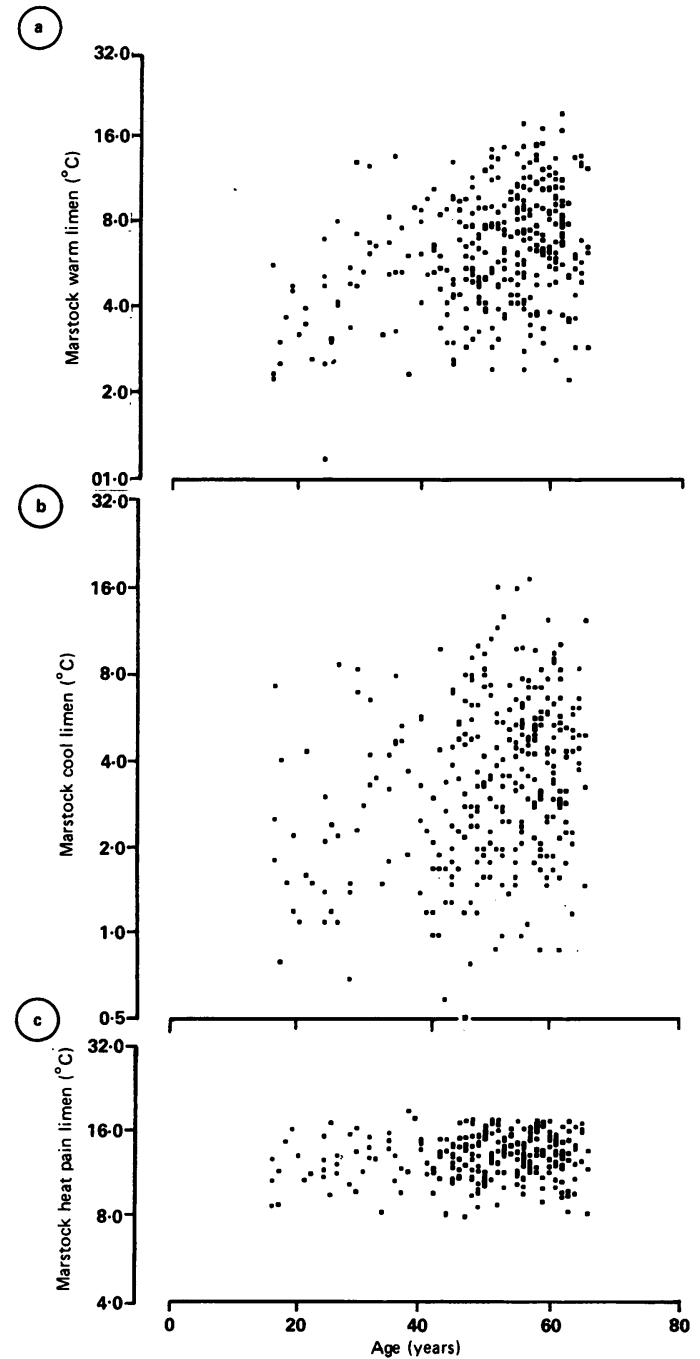

Fig 2 Scattergrams showing the relationships between Marstock thresholds and age in diabetic patients. For comparative purposes, the thresholds are represented using the same logarithmic scales. (a) Warm threshold. $r=0.30$ (367), $p<0.001$. (b) Cool threshold. $r=0.18$ (357). $p=0.001$. (c) Heat pain threshold. $r=0.13$ (299), $p=0.03 .11$ patients did not feel thermal pain at the $50^{\circ} \mathrm{C}$ safety cut-out temperature.

\section{(2) Diabetic subjects}

(a) Sensortek method Mean thermal threshold was $1.00 \mathrm{SD} 1.08^{\circ} \mathrm{C}$. In the larger number of diabetic subjects studied, the correlation of thermal thresholds with age achieved statistical significance $(r=0.25$ (576), $\mathrm{p}<0.001$ ), figure 1 , though the increase in threshold per decade $\left(0.13^{\circ} \mathrm{C}\right)$ is small. The difference in mean thermal thresholds between males and

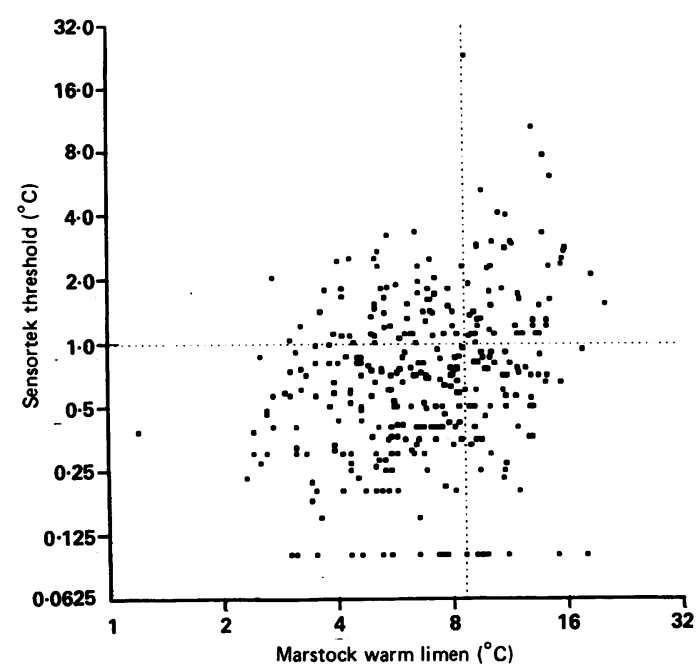

Fig 3 Scattergram of Marstock warm and Sensortek thresholds in 367 diabetic subjects. Both thresholds are shown on a logarithmic scale. The crossed dotted lines represent upper $95 \%$ confidence intervals established from normal subjects $\left(0.98^{\circ} \mathrm{C}\right.$ for Sensortek method, $8.6^{\circ} \mathrm{C}$ for Marstock warm thresholds). $r=0.22, p<0.001$.

females was just significant $\left(1 \cdot 19 \mathrm{SD} 1.89^{\circ} \mathrm{C}\right.$ vs. 0.89 SD $0.98^{\circ} \mathrm{C}, \mathrm{p}=0.05$ ), and there was a significant association between threshold and weight $(\mathrm{r}=0.18$ (194), $p=0.01)$, but not height $(r=0.10$ (184), $\mathrm{p}=0 \cdot 17$ ). Multivariate analysis showed that the duration of diabetes was significantly associated with Sensortek thresholds (2-tail p value 0.04).

(b) Marstock method All thresholds also showed small but significant increases with age (warm $r=0.30$ (367), $p<0.001 ;$ cool $r=0.18$ (357), $p=0.001$; heat pain $r=0.13(299), p=0.03)$. The ${ }^{\circ} \mathrm{C}$ changes per decade were: warm threshold $0.8^{\circ} \mathrm{C}$, cool threshold $0.5^{\circ} \mathrm{C}$, and heat pain $0.3^{\circ} \mathrm{C}$. Changes in warm, cool and heat pain thresholds with age are shown in figure 2(a)-(c). Warm and cool thresholds were significantly associated $(r=0.45 \quad$ (367), $\mathrm{p}=0.002$ ) as were warm thresholds and heat pain thresholds $(r=0.33(300), p<0.001)$. Known duration of diabetes did not correlate with the thresholds. Warm thresholds were significantly higher in men than in women $\left(7.7 \mathrm{SD} 3.5^{\circ} \mathrm{C}(214)\right.$ vs. $6.8 \mathrm{SD} 3.2^{\circ} \mathrm{C}$ (128), $\mathrm{p}=0.02$ ) but not cool or heat pain thresholds (4.3 SD $3.0^{\circ} \mathrm{C}(210)$ vs. 3.8 SD 2.4 (125), $\mathrm{p}=0.09 ; 14.5$ SD $5 \cdot 1^{\circ} \mathrm{C}(176) v s .14 \cdot 1$ SD $\left.3 \cdot 1^{\circ} \mathrm{C}(101), \mathrm{p}=0.7\right)$.

(c) Comparison of Sensortek and Marstock methods All Marstock thresholds showed weak but statistically significant associations with the Sensortek threshold (warm $r=0.22$ (367), $p<0.001$; cool $r=0.21$ (358), $\mathrm{p}<0.001$; heat pain $\mathrm{r}=0.23(299), \mathrm{p}<0.001)$. The relationship between the Somedic warm and Sensor- 


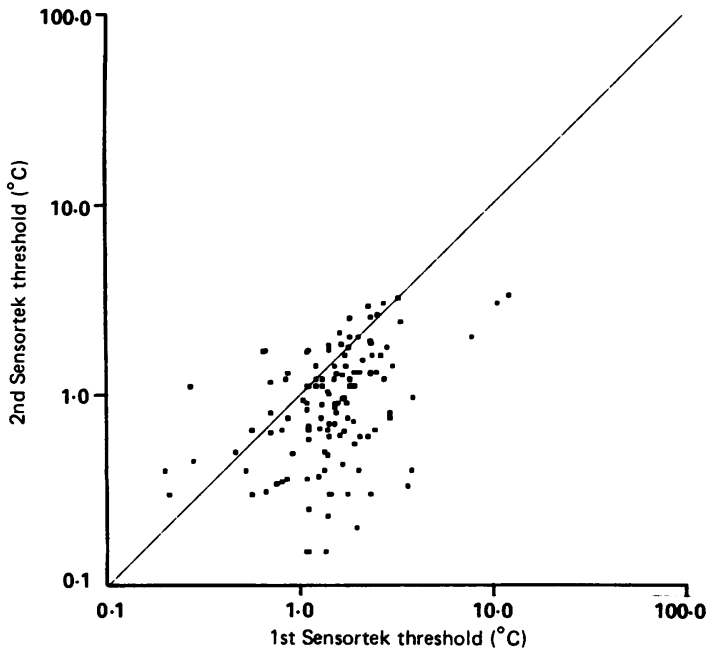

Fig 4 Scattergram showing results of repeat thermal testing in 131 diabetic subjects 1-12 weeks apart. The line of identity is shown. $106(81 \%)$ of patients had lower thermal thresholds on their second visit; the group change is marked and statistically significant: visit $11.75 S D 1.51^{\circ} \mathrm{C}$, visit 21.04 $S D 0.71^{\circ} \mathrm{C}, p<0.001$.

tek thresholds is shown in figure 3 . The crossed dotted lines indicate the upper limits of normal (mean +2 SD) for the two methods. The two methods were concordant (that is, both results normal or abnormal) in only $65 \%$ of patients. The proportion of patients with abnormal thresholds was similar for the Sensortek and for Marstock warm and cool thresholds $(32.5 \%, 31.9 \%$ and $33.9 \%$ respectively). The prevalence of abnormal heat pain thresholds was lower, $14.3 \%$. Likewise, similar proportions of patients had an abnormal Sensortek and Marstock threshold (abnormal Sensortek and Marstock warm $15.2 \%$, abnormal Sensortek and Marstock cool $18.4 \%$ ). Abnormal Sensortek and heat pain thresholds were found in only $7.9 \%$ of patients.

(d) Repeat thermal testing Figure 4 shows the results of repeat Sensortek thermal testing in 131 patients. There was a significant fall in thermal thresholds at the second visit $\left(1.75 \mathrm{SD} 1.51^{\circ} \mathrm{C} v \mathrm{~s} .1 .04\right.$ SD $0.71^{\circ} \mathrm{C}, \mathrm{p}<0.001$ ).

(e) Patients with symptomatic neuropathy 128 patients had symptomatic neuropathy. They were neither older nor were known to have been diabetic for longer than those without symptoms (52.1 SD $10 \cdot 6 \mathrm{vs}$. 50.2 SD 11.4 years; 8.3 SD 7.6 vs. 7.9 SD 8.2 years). However, they had higher Sensortek thresholds $(1.26$ SD $1.26^{\circ} \mathrm{C}$ vs. $0.89 \mathrm{SD} 1.7^{\circ} \mathrm{C}, \mathrm{p}=0.04$ ), and all the Marstock thresholds were significantly elevated (warm $8.4 \mathrm{SD} 3.9^{\circ} \mathrm{C} v s .6 .8 \mathrm{SD} 2.9^{\circ} \mathrm{C}, \mathrm{p}<0.001$; cool 4.6 SD $3.0^{\circ} \mathrm{C} v s .3 .9 \mathrm{SD} 2.6^{\circ} \mathrm{C}, \mathrm{p}=0.03$; heat pain 15.2 SD $5.9^{\circ} \mathrm{C}$ vs. $13.7 \mathrm{SD} 3.0^{\circ} \mathrm{C}, \mathrm{p}=0.01$ ).

\section{Discussion}

This is the first comparative study of two devices employing different psychophysical algorithms for measuring thermal sensitivity. Thresholds measured by both methods are statistically significantly associated when large numbers of patients are tested. The prevalence of abnormality detected by each method is similar, though thermal pain thresholds, which may be measuring function in a different population of neurons ${ }^{10}$ are less frequently abnormal. Our data in normal subjects show that both methods have high intrapatient variability, similar to that found for other psychophysical methods. " Limited experience with other thermal testing apparatus, for example the computerised forced-choice device described by Jamal $e t a l^{12}$ and the method of Fowler $e t$ $a l,{ }^{13}$ suggests that they have similar intrasubject variability to the methods used in the current study. ${ }^{14}$

Both devices take account of some of the peripheral stimulation factors known to affect thermal thresholds when measured in physiological laboratories using strict environmental control and normal subjects welltrained in thermal discrimination. ${ }^{1516}$ Their relevance in the clinical setting with a naive and neuropathic population is not known. Nevertheless, we have wherever possible, incorporated controllable stimulus-related factors, including using the same test site, in order to avoid errors caused by differences in skin thickness, ${ }^{17}$ and maintaining a constant basal skin temperature within the zone of thermal neutrality, between 30 and $32^{\circ} \mathrm{C} .^{18}$

For both pieces of apparatus the remaining stimulus-related factors are either similar or have not been taken into account. Increasing the thermode surface area above $8 \mathrm{~cm}^{2}$ has a negligible effect on thermal threshold, ${ }^{15}$ and all thermodes for testing limbs now have surface areas between 12.5 and $26 \mathrm{~cm}^{2}$. Neither device takes account of thermode application pressure. Its importance in clinical testing has not been systematically investigated, though some authors have attempted to control for it when designing their apparatus. ${ }^{41219}$ Experimental evidence ${ }^{20}$ suggests that within a physiological temperature range thermoreceptors are not activated by tactile or vibratory stimuli. Pressure effects are unlikely to be as important in thermal threshold testing as in vibration testing. ${ }^{21}$

The major differences between these two pieces of apparatus are therefore the nature of the thermal stimulus (static or dynamic) and the psychophysical algorithm used to calculate threshold. In experimental work the importance of the choice of algorithm is well recognised. A forced-choice algorithm, usually accompanied by a variant of the up-down-transformation rule (UDTR), is theoretically preferable. However, our results suggest that in clinical use the variability of 
a forced-choice UDTR method (Sensortek) is similar to one employing the method of limits (Marstock). The reasons for this are not clear, but it may be that any improved precision of a forced-choice method may be negated by the much longer test time. Our results support previous suggestions ${ }^{11}$ that the most important factor in the variability of all thermal threshold measurements is the central processing factors.

There is a significant reduction in Sensortek thermal threshold on repeat testing. We do not know whether this change depends on the psychophysical algorithm, the static nature of the stimuli, or whether it is characteristic of thermal testing in general. However, we have found that the phenomenon does not occur on repeat vibration threshold testing with the Ohio Biothesiometer (method of limits), and Bertlesmann et $\mathrm{al}^{4}$ did not find the same phenomenon in a small number of diabetic patients tested with his static thermal discrimination device. This suggests that there is a prolonged learning effect in some forced-choice methods which may lead to a significant improvement in performance in inexperienced subjects or operators. It is likely that the learning process is quicker with a method of limits, though our results suggest that it is not completed within the first 10 warm stimuli using the Marstock method.

As in other studies with the Sensortek apparatus, we found no significant change in thermal sensitivity with age in a group of 78 normal subjects. ${ }^{822}$ The age effect is therefore smaller than with the Marstock apparatus where, in the same group, there was a small but statistically significant increase in all three thresholds with age. A deterioration with age in thermal sensitivity in diabetics has been noted with all thermal threshold methods, though it is much less marked than the age-related changes in vibration thresholds ${ }^{2123}$ or cardiovascular vagal autonomic function ${ }^{24}$ and small in relation to the variability of the methods. Thermal thresholds measured by either apparatus therefore do not require age-correction. For both methods, females had lower thermal thresholds than males, though Marstock cool and heat pain thresholds were similar. Gender differences, probably related to differences in height, weight, skin thickness and adiposity, are more pronounced in tests of large-fibre function (vibration sensation and electrophysiology). ${ }^{25}$ Patients with symptoms had higher thermal and heat pain thresholds; other studies have shown similar results. ${ }^{46}$ Abnormal nociception in diabetics is complex ${ }^{27}$ and the gross subjective response measured here is unlikely to be sensitive to subtle changes in the pain pathways.

It is not known whether "warm" or "cool" fibres are preferentially affected in diabetic neuropathy, though the nerve fibre populations for warm and cool sensation are undoubtedly different. ${ }^{2829}$ Clinical studies in peripheral neuropathies provide conflicting evidence of differential involvement. The current study indicates that the prevalence of abnormal warm and cool thresholds using the Marstock device is similar and supports the view that diabetic neuropathy frequently involves a variety of nerve fibre types. ${ }^{22} 30$ Jamal et al,,$^{12}$ using a strict forced-choice method, also found similar prevalence rates of abnormal warm and cool thresholds in patients with peripheral neuropathies. However, Sosenko et al ${ }^{30}$ using a modification of the Sensortek method to measure separately warm and cool thresholds, found that warm insensitivity was more prevalent than cool, particularly if the patients were in poor diabetic control. These divergent results probably arise from the use of different devices under different experimental conditions.

What factors, then, should be taken into account when choosing a thermal threshold testing device for clinical use, and is a single method sufficient? The most important requirements are for a screening method that is simple both for the subject and the operator, one that can be calibrated and standardised for multicentre use and one that is robust and cheap. Many of these requirements are fulfilled by the Marstock device and its modification. Although thresholds measured with it are higher than those obtained using a forcedchoice method (whether the stimuli are static or dynamic), it is likely that this is due to the rapid changes of thermode temperature and the associated patient response times. It should not be regarded as a major objection in clinical practice. Much of the basic research on thermal threshold measurement carried out by Kenshalo ${ }^{1518}$ used a method of limits to yield "absolute" thermal thresholds, by using very slowly changing thermode temperatures. Allowing for the marked inter- and intra-patient variability of the methods, we have shown broadly concordant results for both a static and a dynamic measurement of thermal sensitivity and they cannot therefore be regarded as independent measures. Thermal testing by a single method is therefore sufficient for routine clinical screening.

The popularity of sensory threshold testing owes much to the simplicity of the tests, which can be carried out by non-specialists. However, the true role of thermal threshold testing in diabetic neuropathy, particularly in relation to other methods of assessment, has yet to be firmly established. Thermal threshold testing assesses not only the integrity of peripheral small fibres, but a neurological pathway extending from peripheral receptor to the cerebral cortex. Other, more objective measures of localised peripheral small fibre function are required and have been described. ${ }^{31}$ They are currently research rather than clinical tools, but it is likely that when they have been simplified such tests will be preferable. At the 
moment, however, semi-objective psychophysical thermal threshold testing is the only method routinely available for peripheral small fibre testing. Our results suggest that when a thermal threshold method is employed, the precision and reproducibility of the test is related neither to the apparatus nor to the psychophysical algorithm it uses.

We thank Dr S McHardy-Young and Dr E M Jepson for allowing us to study their patients and Pfizer (UK) for the Sensortek apparatus.

\section{References}

1 Dorfman LJ, Cummins KL, Reaven GM, Ceranski J, Greenfield MS, Doherne L. Studies of diabetic polyneuropathy using conduction velocity distribution (DCV) analysis. Neurology 1983;33:773-9.

2 Lawrence RD. The diabetic life: a concise practical manual. 17th edition. London: Churchill 1965:157.

3 Guy RJC, Clark CA, Malcolm PN, Watkins PJ. Evaluation of thermal and vibration sensation in diabetic neuropathy. Diabetologia 1985;28:131-7.

4 Bertlesmann FW, Heimans JJ, Weber EJM, et al. Thermal discrimination thresholds in normal subjects and in patients with diabetic neuropathy. J Neurol Neurosurg Psych 1985; 48:686-90.

5 Fruhstorfer H, Lindblom U, Schmidt WG. Method for quantitative estimation of thermal thresholds in patients. $J$ Neurol Neurosurg Psych 1976;39:1071-5.

6 Dyck PJ, Zimmerman IR, O'Brien PC, et al. Introduction of automated systems to evaluate touch-pressure, vibration, and thermal cutaneous sensation in man. Ann Neurol 1978;4:502-10.

7 Sekuler R, Nash DN, Armstrong R. Sensitive, objective procedure for evaluating response to light touch. Neurology (Minneap) 1973;23:1282-91.

8 Arezzo JC, Schaumburg HH, Laudadio C. Thermal sensitivity tester: device for quantitative assessment of thermal sense in diabetic neuropathy. Diabetes 1986;35:590-2.

9 Wetherhill GB, Chen H, Vasudeva RB. Sequential estimation of quantal response curves: a new method of estimation. Biometrika 1966;53:439-54.

10 Consensus statement: report and recommendations of the San Antonio conference on diabetic neuropathy. Diabetes Care 1988;11:592-7.

11 Fagius J, Wahren LK. Variability of sensory threshold determination in clinical use. J Neurol Sci 1981;51:11-27.

12 Jamal GA, Hansen S, Weir AI, Ballantyne JP. An improved automated method for the measurement of thermal thresholds. 1. normal subjects. $J$ Neurol Neurosurg Psych 1985;48:354-60.

13 Fowler CJ, Carroll MB, Burns D, Howe N, Robinson K. A portable system for measuring cutaneous thresholds for warming and cooling. J Neurol Neurosurg Psych 1987;50:1211-5.

14 Levy DM, Abraham RR, Abraham RM. A comparison of four methods for measuring thermal thresholds in diabetes. Diabetic Med 1987;4:591A.

15 Kenshalo DR. Psychophysical studies of temperature sensitivity. In: Neff WD, ed. Contributions to sensory physiology. Vol 4. New York, London: Academic Press, 1970:19-74.

16 Lele PP. Relationship between cutaneous thermal thresholds, skin temperature and cross-sectional area of the stimulus. J Physiol (Lond) 1954;126:191-205.

17 Stoll AM. Thermal properties of human skin related to nondestructive measurement of epidermal thickness. $J$ Invest Dermatol 1977;69:328-32.

18 Kenshalo DR, Scott HA Jr. Temporal course of thermal adaptation. Science 1966;151:1095-6.

19 Jamal GA, Weir AI, Ballantyne JP, Hansen S. Thermal discrimination thresholds in normal subjects and in patients with diabetic neuropathy. J Neurol Neurosurg Psych 1986;49:335-6.

20 Konietzny F, Hensel $\mathrm{H}$. Warm fiber activity in human skin nerves. Pflügers Arch 1975;359:265-7.

21 Goldberg JM, Lindblom U. Standardised method of determining vibratory perception thresholds for diagnosis and screening in neurological investigation. $J$ Neurol Neurosurg Psych 1979;42:793-803.

22 Levy DM, Abraham RR, Abraham RM. Small- and large-fiber involvement in early diabetic neuropathy: a study with the medial plantar response and sensory thresholds. Diabetes Care 1987;10:441-7.

23 Bloom S, Till S, Sonksen P, Smith S. Use of a biothesiometer to measure individual thresholds and their variation in 519 nondiabetic subjects. Br Med J 1984;288:1793-5.

24 Smith SA. Reduced sinus arrhythmia in diabetic autonomic neuropathy: the value of an age-related normal range. $\mathrm{Br} M e d \mathrm{~J}$ 1982;285:1599-601.

25 Abraham RR, Levy DM. Diabetic neuropathy: measurement and quantitation. In: Ward JD, ed. Diabetic neuropathy. Proceedings of a satellite symposium of the 13th IDF Congress, 1988. London: John Wiley, 1989, in press.

26 Ziegler D, Mayer P, Wiefels K, Gries FA. Assessment of small and large fiber function in long-term type 1 (insulin-dependent) diabetic patients with and without painful neuropathy. Pain 1988;34:1-10.

27 Morley GK, Mooradian AD, Levine AS, Morley JE. Mechanism of pain in diabetic peripheral neuropathy: effect of glucose on pain perception in humans. Am J Med 1984;77:79-82.

28 Fowler CJ, Sitzoglou K, Ali Z, Halonen P. The conduction velocities of peripheral nerve fibres conveying sensations of warming and cooling. J Neurol Neurosurg Psych 1988;51: $1164-70$.

29 Levy DM, Abraham RR, McHardy-Young S, Reid G, Gilbert D, Abraham RM. Abnormal thermal sensation in diabetes reflects abnormalities in heat pain sensation, autonomic function and large fibre tests. Diabetes Res Clin Pract 1988;5(Suppl 1):S436.

30 Sosenko JM, Kato M, Soto RA, Gadia MT, Ayyar DR. Specific assessments of warm and cool sensitivities in adult diabetic patients. Diabetes Care 1988;11:481-3.

31 Parkhouse N, LeQuesne PM. Quantitative objective assessment of peripheral nociceptive C fibre function. J Neurol Neurosurg Psych 1988;51:28-34. 